\title{
Study on an N-parallel FENE-P constitutive model based on multiple relaxation times for viscoelastic fluid
}

\author{
Jingfa Li ${ }^{1,2[0000-0002-5207-1156]}$, Bo Yu ${ }^{1[0000-0002-4231-6914]}$, Shuyu Sun ${ }^{2[0000-0002-3078-864 X]}$, \\ Dongliang Sun ${ }^{1[0000-0002-6253-0416] ~}$ \\ ${ }^{1}$ School of Mechanical Engineering, Beijing Key Laboratory of Pipeline Critical Technology \\ and Equipment for Deepwater Oil \& Gas Development, Beijing Institute of Petrochemical \\ Technology, Beijing 102617, China \\ ${ }^{2}$ Computational Transport Phenomena Laboratory, Division of Physical Science and Engineer- \\ ing, King Abdullah University of Science and Technology, Thuwal 23955-6900, Saudi Arabia \\ yubobox@vip.163.com; shuyu.sun@kaust.edu.sa
}

\begin{abstract}
An N-parallel FENE-P constitutive model based on multiple relaxation times is proposed in this paper, which aims at accurately describing the apparent viscosity of viscoelastic fluid. The establishment of N-parallel FENE-P constitutive model and the numerical approach to calculate the apparent viscosity are presented in detail, respectively. To validate the performance of the proposed constitutive model, it is compared with the conventional FENE-P constitutive model (It only has single relaxation time) in estimating the apparent viscosity of two common viscoelastic fluids: polymer and surfactant solutions. The comparative results indicate the N-parallel FENE-P constitutive model can represent the apparent viscosity of polymer solutions more accurate than the traditional model in the whole range of shear rate $\left(0.1 \mathrm{~s}^{-1} \sim 1000 \mathrm{~s}^{-1}\right)$, and the advantage is more noteworthy especially when the shear rate is higher $\left(10 \mathrm{~s}^{-1} \sim 1000 \mathrm{~s}^{-1}\right)$. Despite both the proposed model and the traditional model can't capture the interesting shear thickening behavior of surfactant solutions, the proposed constitutive model still possesses advantage over the traditional one in depicting the apparent viscosity and first normal stress difference. In addition, the N-parallel FENE-P constitutive model demonstrates a better applicability and favorable adjustability of the model parameters.
\end{abstract}

Keywords: FENE-P Constitutive Model, N-Parallel, Viscoelastic Fluids, Multiple Relaxation Times, Apparent Viscosity

\section{Introduction}

In 1948, Toms [1] first reported an interesting phenomenon that adding a little bit of additive, such as some kinds of polymer, into the turbulent flows would induce a drag reduction (DR) obviously in the 1st International Rheology Congress, it was later called turbulent DR effect or Toms' effect. From the 1950 to now, as two kinds of successful turbulent DR additives, the researches on turbulent DR mechanism and industrial applications of the polymer and surfactant attract a multitude of scholars' attentions. 
Among the research approaches, numerical simulation has become a significant tool to get insight into the DR mechanism of viscoelastic fluid with the rapid development of computer science in recent years. However, distinguishing from Newtonian fluid, the viscoelastic fluid shows complicated rheological properties and elastic effect. It is a prerequisite to build a constitutive model to describe the quantitative relation between elastic stress and strain in the numerical simulation of turbulent DR of viscoelastic fluids. In general, the upper convected Maxwell (UCM) model [2], Oldroyd-B model [3, 4] and Giesekus model [5] as well as FENE-P model [6] are commonly used constitutive models in a large number of literatures. For the desirable performance to represent the shear-thinning phenomenon compared with the UCM model and Oldroyd-B models, the Giesekus and FENE-P models have been applied widely.

It is well known that the rheological properties and turbulent DR effect are closely related to the microstructures (long chain structures for polymer solution or network structures for surfactant solution) formed in the viscoelastic fluids. Therefore, it is necessary to delve into the validity of the constitutive model from the perspective of relaxation-deformation of the microstructures. For the polymer solution, as shown in Fig. 1(a), the long chain structures would exert relaxation-deformation under the shear effect in flow, sometimes they even tangled with each other. Different from polymer solution, the DR effect of surfactant solution depends on the micelles composed of small surfactant molecules. As illustrated in Fig. 1(b), the spherical micells, rod-like micelles and network structures are usually formed in sequence with the increase of concentration under the shear effect in flow, which is also accompanied with relaxation-deformation of microstructures. From above all, it is evident to see that various relaxationdeformations of the microstructures will take place in the viscoelastic fluid under shear effect. In the relaxation-deformation process, the relaxation time is a key parameter for constitutive model. In polymer and surfactant solutions, the relaxation time is referred to the time consumed during a deformation period of the microstructure, it can characterize the strength of elastic effect of the viscoelastic fluid. However, the real viscoelastic fluid has a relaxation time spectrum but not only single relaxation time for the anisotropy caused by different configurations and various deformations of the microstructures under the shear in flow. In the theoretical and experimental researches of the DR effect, the relaxation time spectrum is always simplified as single relaxation time because of the sophisticated analysis when relaxation time spectrum is taken into consideration. Correspondingly, the constitutive models commonly used are characterized with only single relaxation time, which is obviously inconsistent with the actual physical process. It is the main reason responsible for the deviations between the theoretical, experimental and numerical studies of DR mechanism of viscoelastic fluid.

Based on the above analysis of the relaxation-deformation characteristics of the microstructures in viscoelastic fluid, it is apparent that the conventional constitutive models with single relaxation time can not accurately characterize the anisotropy of relaxation-deformation. Therefore, considering the idea of multiple relaxation times and the advantage of FENE-P constitutive model that the deformations are nonlinear, an $\mathrm{N}$ parallel FENE-P constitutive model based on multiple relaxation times is proposed in this study. 


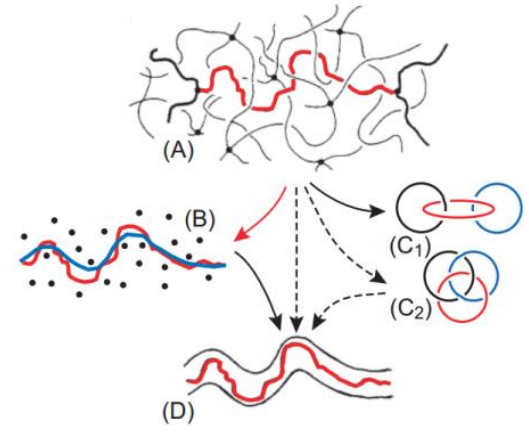

(a) high-molecular polymer solution [7]

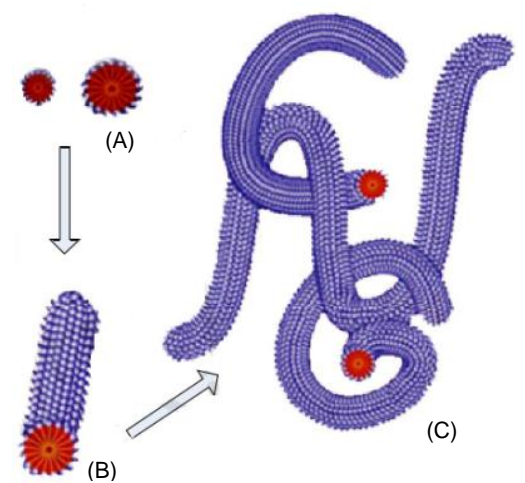

(b) Surfactant solution [8]

Fig. 1. Schematics of microstructures formed in the viscoelastic fluids.

\section{The establishment of $\mathbf{N}$-parallel FENE-P constitutive model based on multiple relaxation times}

In this Section, the drawback of conventional FENE-P constitutive models that only has single relaxation time is taken into account and an N-parallel FENE-P constitutive model based on multiple relaxation times is proposed. The core idea of the proposed constitutive model is to put $N$ FENE-P models in parallel to describe the rheological behaviors of viscoelastic fluids and characterize the anisotropy of relaxation-deformation of the microstructures. The establishment of the proposed N-parallel FENE-P constitutive model is presented detailedly in the following text.

To get insight into the rheological properties of the viscoelastic fluid from the microcosmic perspective, Bird et al [6] modeled the polymer macromolecules by a discrete-element model with finitely extensible nonlinear elastic (FENE) characteristic, which is called spring-dumbbell model too. The FENE-P constitutive model is a member of the FENE model family with Peterlin's approximation [9], and its governing equation reads,

$$
\boldsymbol{\tau}_{\mathrm{v}}+\frac{\lambda}{f(r)} \boldsymbol{\tau}_{\mathrm{v}}^{\nabla}=2 \frac{\eta_{\mathrm{v}}}{f(r)} \boldsymbol{D}
$$

where $\boldsymbol{\tau}_{\mathrm{v}}$ is the extra elastic stress tensor; $\lambda$ is the relaxation time; $f(r)$ is nonlinear factor to ensure the finite extensibility; $\eta_{\mathrm{v}}$ is zero-shear-rate dynamic viscosity; $\boldsymbol{D}$ is the deformation rate tensor, $\boldsymbol{D}=\left(\nabla \boldsymbol{u}+(\nabla \boldsymbol{u})^{\mathrm{T}}\right) / 2$.

As illustrated in Fig. 2, when $N$ FENE-P models are paralleled together, the N-parallel FENE-P constitutive model satisfies the following characteristics:

(1) The extra elastic stress tensor of the N-parallel FENE-P model is equal to the summation of the extra elastic stress tensors of the total $N$ branching FENE-P models, 


$$
\boldsymbol{\tau}_{\mathrm{V}}=\boldsymbol{\tau}_{\mathrm{V} 1}+\boldsymbol{\tau}_{\mathrm{V} 2}+\ldots+\boldsymbol{\tau}_{\mathrm{V} N}=\sum_{i=1}^{N} \boldsymbol{\tau}_{\mathrm{V} i}
$$

(2) The deformation rate tensor of the N-parallel FENE-P model is identical to the deformation rate tensor of each branching FENE-P model,

$$
\boldsymbol{D}=\boldsymbol{D}_{1}=\boldsymbol{D}_{2}=\ldots=\boldsymbol{D}_{N}
$$

where in the above two equations the subscript $N$ denotes the number of the branching FENE-P models, $N \geqslant 2 ; \boldsymbol{\tau}_{\mathrm{V} 1}, \boldsymbol{\tau}_{\mathrm{V} 2}, \ldots, \boldsymbol{\tau}_{\mathrm{V} N}$ are the extra elastic stress tensors of the branching FENE-P models, respectively; $\boldsymbol{D}_{1}, \boldsymbol{D}_{2}, \ldots, \boldsymbol{D}_{N}$ are the deformation rate tensors of the branching FENE-P models, respectively.

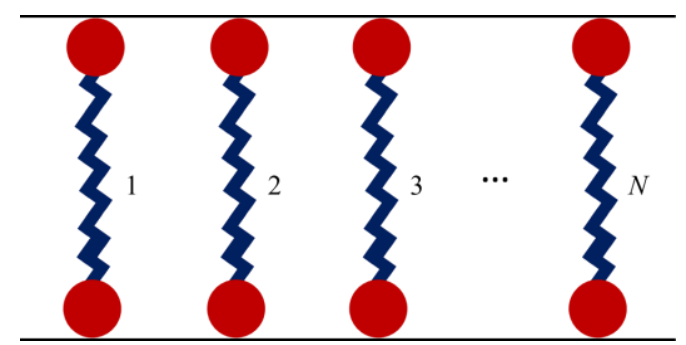

Fig. 2. Schematic of the N-parallel FENE-P model.

The mathematical relation between $\boldsymbol{\tau}_{\mathrm{V} i}$ and $\boldsymbol{D}_{i}$ of the $i$ th branching FENE-P model can be obtained evidently based on Eq. (1),

$$
\boldsymbol{\tau}_{\mathrm{v} i}+\frac{\lambda_{i}}{f\left(r_{i}\right)} \boldsymbol{\tau}_{\mathrm{v} i}^{\nabla}=2 \frac{\eta_{\mathrm{V} i}}{f\left(r_{i}\right)} \boldsymbol{D}_{i}
$$

where $\lambda_{i}, f\left(r_{i}\right)$ respectively represent the relaxation time and nonlinear factor of the $i$ th branching FENE-P model; $\eta_{\mathrm{V} i}$ denotes the zero-shear-rate dynamic viscosity of the $i$ th branching FENE-P model.

Substituting Eqs. (3) (4) into Eq. (2), the N-parallel FENE-P constitutive model can be written as follows,

$$
\sum_{i=1}^{N} \boldsymbol{\tau}_{\mathrm{v}_{i}}+\sum_{i=1}^{N}\left(\frac{\lambda_{i}}{f\left(r_{i}\right)} \boldsymbol{\tau}_{\mathrm{v} i}^{\nabla}\right)=2 \sum_{i=1}^{N} \frac{\eta_{\mathrm{v} i}}{f\left(r_{i}\right)} \boldsymbol{D}
$$

From above equation we can see the N-parallel FENE-P constitutive model is a more general constitutive model. The conventional FENE-P constitutive model with single relaxation time can be regarded as an exception of the N-parallel FENE-P constitutive model when $N=1$.

It is worth noting that the N-parallel FENE-P constitutive model can be rearranged as branching expression form for the convenience of numerical calculation, 


$$
\left\{\begin{array}{c}
\boldsymbol{\tau}_{\mathrm{v} 1}+\frac{\lambda_{1}}{f\left(r_{1}\right)} \boldsymbol{\tau}_{\mathrm{v} 1}^{\nabla}=2 \frac{\eta_{\mathrm{v} 1}}{f\left(r_{1}\right)} \boldsymbol{D} \\
\boldsymbol{\tau}_{\mathrm{v} 2}+\frac{\lambda_{2}}{f\left(r_{2}\right)} \boldsymbol{\tau}_{\mathrm{v} 2}^{\nabla}=2 \frac{\eta_{\mathrm{v} 2}}{f\left(r_{2}\right)} \boldsymbol{D} \\
\cdot \\
\cdot \cdot \frac{\lambda_{i}}{f\left(r_{i}\right)} \boldsymbol{\tau}_{\mathrm{v} i}^{\nabla}=2 \frac{\eta_{\mathrm{v} i}}{f\left(r_{i}\right)} \boldsymbol{D} \\
\boldsymbol{\tau}_{\mathrm{v} i}+ \\
\boldsymbol{\tau}_{\mathrm{v} N}+\frac{\lambda_{N}}{f\left(r_{N}\right)} \boldsymbol{\tau}_{\mathrm{v} N}^{\nabla}=2 \frac{\eta_{\mathrm{v} N}}{f\left(r_{N}\right)} \boldsymbol{D}
\end{array}\right.
$$

\section{Numerical approach to obtain the apparent viscosity by solving the $\mathbf{N}$-parallel FENE-P constitutive model}

Apparent viscosity is one of the most significant parameters to measure the rheological properties of viscoelastic fluid. However, a large body of literature reveal that the conventional constitutive model with single relaxation time show unfavorable performance in describing the apparent viscosity compared with experimental results [10]. In order to validate whether the proposed N-parallel FENE-P constitutive model gains advantage over the traditional one in representing the rheological properties of viscoelastic fluid, the numerical approach to calculate the apparent viscosity by solving the $\mathrm{N}$ parallel FENE-P constitutive model is introduced in this Section.

Under a certain temperature and solution concentration, the viscosity of viscoelastic fluid is called apparent viscosity or shear viscosity for the reason that it is not a constant but has close relation to the shear rate. Generally, the apparent viscosity reads,

$$
\eta_{a}=\tau_{x y} / \dot{\gamma}=\left(\tau_{\mathrm{N}, x y}+\sum_{i=1}^{N} \tau_{\mathrm{v} i, x y}\right) / \dot{\gamma}
$$

where $\eta_{a}$ represents the apparent viscosity; $\dot{\gamma}$ denotes the shear rate; $\tau_{\mathrm{N}, x y}, \sum_{i=1}^{N} \tau_{\mathrm{v} i, x y}$ denote the extra viscous stress contributed by solvent and solute, respectively.

It can be seen from Eq. (7) that it is a prerequisite to obtain the mathematical relation between $\sum_{i=1}^{N} \tau_{\mathrm{v} i, x y}$ and $\dot{\gamma}$ before calculating the apparent viscosity. In general, water is 
a commonly used solvent in viscoelastic fluid and its apparent viscosity can be approximately regarded as constant under a certain temperature and solution concentration. Thus the terms $\tau_{\mathrm{N}, x y}$ and $\dot{\gamma}$ satisfy the following relation,

$$
\tau_{\mathrm{N}, x y}=\eta_{\mathrm{N}} \dot{\gamma}
$$

where $\eta_{\mathrm{N}}$ denotes the dynamic viscosity of solvent.

As two successfully used drag-reducing additives, the viscosities of polymer and surfactant change remarkably with the variation of shear rate. At certain temperature and solution concentration, the $\sum_{i=1}^{N} \tau_{\mathrm{v} i, x y}$ and $\dot{\gamma}$ meet the below equation,

$$
\sum_{i=1}^{N} \tau_{\mathrm{v} i, x y}=\sum_{i=1}^{N} \eta_{\mathrm{v} i}(\dot{\gamma}) \dot{\gamma}
$$

where $\eta_{\mathrm{v} i}(\dot{\gamma})$ represents the dynamic viscosity of solute.

Substituting Eqs. (8) (9) into Eq. (7), the expression of apparent viscosity of viscoelastic fluid can be obtained as below,

$$
\eta_{a}=\eta_{\mathrm{N}}+\sum_{i=1}^{N} \eta_{\mathrm{v} i}(\dot{\gamma})
$$

Therefore, the $\sum_{i=1}^{N} \tau_{\mathrm{v} i, x y}$ should be first computed from Eq. (6) to get $\sum_{i=1}^{N} \eta_{\mathrm{v} i}(\dot{\gamma})$. For the sake of concision but without loss of generality, the double-parallel FENE-P constitutive model is taken as an example to represent N-parallel FENE-P constitutive model in illustrating the numerical approach to calculate apparent viscosity. For the convenience, the present study focuses on the two-dimensional simple shear flow. Based on the above assumptions, the Eq. (6) and Eq. (10) can be simplified as,

$$
\left\{\begin{array}{c}
\tau_{\mathrm{v} 1, x x}+\tau_{\mathrm{v} 2, x x}+\frac{\lambda_{1}}{f\left(r_{1}\right)}\left(\frac{\partial \tau_{\mathrm{v} 1, x x}}{\partial t}-2 \frac{\partial u}{\partial y} \tau_{\mathrm{v} 1, x y}\right)+\frac{\lambda_{2}}{f\left(r_{2}\right)}\left(\frac{\partial \tau_{\mathrm{v} 2, x x}}{\partial t}-2 \frac{\partial u}{\partial y} \tau_{\mathrm{v} 2, x y}\right)=0 \\
\tau_{\mathrm{v} 1, y y}+\tau_{\mathrm{v} 2, y y}+\frac{\lambda_{1}}{f\left(r_{1}\right)}\left(\frac{\partial \tau_{\mathrm{v} 1, y y}}{\partial t}\right)+\frac{\lambda_{2}}{f\left(r_{2}\right)}\left(\frac{\partial \tau_{\mathrm{v} 2, y y}}{\partial t}\right)=0 \\
\tau_{\mathrm{v} 1, x y}+\tau_{\mathrm{v} 2, x y}+\frac{\lambda_{1}}{f\left(r_{1}\right)}\left(\frac{\partial \tau_{\mathrm{v} 1, x y}}{\partial t}-\tau_{\mathrm{v} 1, y y} \frac{\partial u}{\partial y}\right)+\frac{\lambda_{2}}{f\left(r_{2}\right)}\left(\frac{\partial \tau_{\mathrm{v} 2, x y}}{\partial t}-\tau_{\mathrm{v} 2, y y} \frac{\partial u}{\partial y}\right) \\
=\frac{\eta_{\mathrm{v} 1}}{f\left(r_{1}\right)}\left(\frac{\partial u}{\partial y}\right)+\frac{\eta_{\mathrm{v} 2}}{f\left(r_{2}\right)}\left(\frac{\partial u}{\partial y}\right) \\
\eta_{a}=\eta_{\mathrm{N}}+\eta_{\mathrm{v} 1}(\dot{\gamma})+\eta_{\mathrm{v} 2}(\dot{\gamma})
\end{array}\right.
$$

For two-dimensional simple shear flow, the shear rate $\dot{\gamma}$ can be expressed as, 


$$
\dot{\gamma}=\frac{\partial u}{\partial y}
$$

It can be easily found that the key step to solve Eq. (11) is to discretize the unsteady term of extra elastic stress. In this paper, the second-order Adams-Bashforth scheme is adopted to discretize the term as below,

$$
\frac{\tau^{n+1}-\tau^{n}}{\Delta t}=\frac{3}{2} F(\tau)^{n}-\frac{1}{2} F(\tau)^{n-1}
$$

Then the Eq. (11) can be discretized as follows based on Eq. (14),

$$
\begin{aligned}
& \tau_{\mathrm{v}_{i, x x}^{n+1}}^{n}=\tau_{\mathrm{v} i, x x}^{n}+\frac{3}{2} \Delta t\left[2 \dot{\gamma} \tau_{\mathrm{v} i, x y}^{n}-\frac{f\left(r_{i}\right)^{n}}{\lambda_{i}} \tau_{\mathrm{v}, x x}^{n}\right]- \\
& \frac{1}{2} \Delta t\left[2 \dot{\gamma} \tau_{\mathrm{vi}, x y}^{n-1}-\frac{f\left(r_{i}\right)^{n-1}}{\lambda_{i}} \tau_{\mathrm{v} i, x x}^{n-1}\right] \\
& \left\{\tau_{\mathrm{v}_{i, y y}^{n+1}}=\tau_{\mathrm{v} i, y y}^{n}-\frac{3}{2} \Delta t\left[\frac{f\left(r_{i}\right)^{n}}{\lambda_{i}} \tau_{\mathrm{v} i, y y}^{n}\right]+\frac{1}{2} \Delta t\left[\frac{f\left(r_{i}\right)^{n-1}}{\lambda_{i}} \tau_{\mathrm{v}_{i, y y}^{n-1}}\right]\right. \\
& \begin{array}{r}
\tau_{\mathrm{v}, i, y}^{n+1}=\tau_{\mathrm{v}, x y}^{n}+\frac{3}{2} \Delta t\left[\dot{\gamma} \tau_{\mathrm{v}, y, y}^{n}-\frac{f\left(r_{i}\right)^{n}}{\lambda_{i}} \tau_{\mathrm{v}, x y}^{n}+\right. \\
\frac{1}{2} \Delta t\left[\dot{\gamma} \tau_{\mathrm{v} i, y y}^{n-1}-\frac{f\left(r_{i}\right)^{n-1}}{\lambda_{i}} \tau_{\mathrm{v} i, x y}^{n-1}+\frac{\eta_{\mathrm{v} i}}{\lambda_{i}} \dot{\gamma}\right]
\end{array}
\end{aligned}
$$

where the superscript $n$ represents time layer; the subscript $i$ denotes the $i$ th branching FENE-P model.

The nonlinear factor $f(r)$ in Eq. (15) is a function of conformation tensor. The trace of conformation tensor should be calculated first before calculating $f(r)$,

$$
\operatorname{trace}\left(c_{\mathrm{v}_{i}}\right)=c_{\mathrm{v}_{i, x x}^{n+1}}+c_{\mathrm{v}_{i, y y}^{n+1}}^{n+1}
$$

where the conformation tensor components $c_{\mathrm{V} i, x x}^{n+1}$ and $c_{\mathrm{v} i, y y}^{n+1}$ are given as below,

$$
\left\{\begin{array}{l}
c_{\mathrm{v} i, x x}^{n+1}=\left(\frac{\lambda_{i}}{\eta_{\mathrm{v} i}} \tau_{\mathrm{v}_{i, x x}^{n+1}}^{n+1}\right) / f\left(r_{i}\right)^{n} \\
c_{\mathrm{v}^{n}, y y}^{n+1}=\left(\frac{\lambda_{i}}{\eta_{\mathrm{v} i}} \tau_{\mathrm{v}_{i, y y}^{n+1}}^{n}+1\right) / f\left(r_{i}\right)^{n}
\end{array}\right.
$$

By solving Eqs. (16) (17), the nonlinear factor of each branching FENE-P constitutive model can be obtained as,

$$
f\left(r_{i}\right)^{n+1}=\frac{L^{2}-3}{L^{2}-\operatorname{trace}\left(c_{\mathrm{v}_{i}}\right)}
$$


where $L$ is the maximum length of polymer molecules or surfactant micelles scaled with its equilibrium value, $L$ is set as 100 in this study.

Hereafter we can calculate the apparent viscosity by solving the N-parallel FENE-P constitutive model based on Eqs. (12) (18). Taking the double-parallel FENE-P constitutive model as an example, the flow chart to calculate the apparent viscosity is shown in Fig. 3.

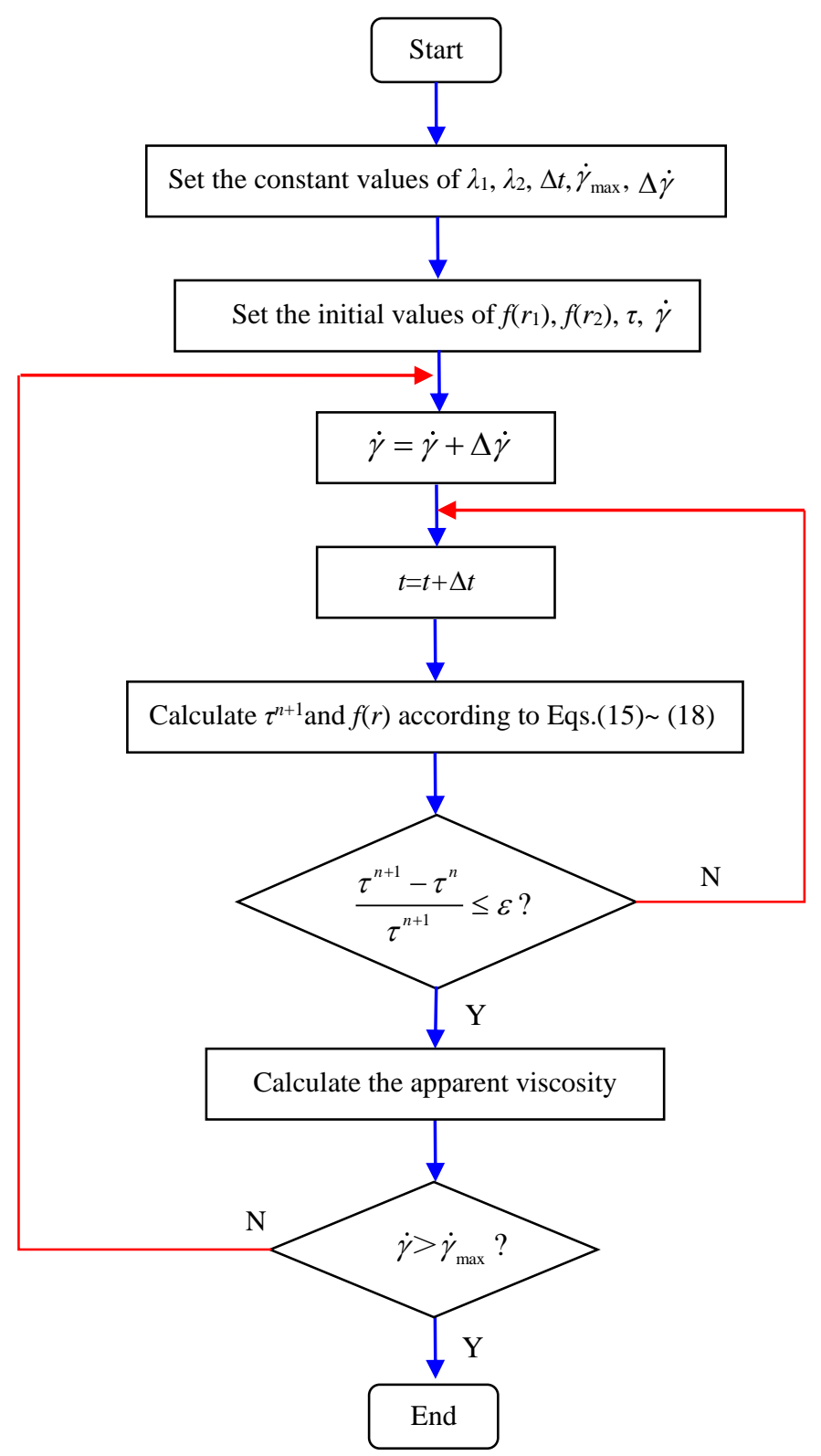


Fig. 3. Flow chart of the numerical calculation of apparent viscosity

\section{Validation of the N-parallel FENE-P constitutive model and results discussion}

\subsection{Determination of model parameters}

Before calculating apparent viscosity by using the N-parallel FENE-P constitutive model, the model parameters, such as relaxation time $\lambda_{i}$ and dynamic viscosity $\eta_{\mathrm{V} i}$, should be determined or given first. For the sake of following discussion, we introduce the parameter $\beta_{i}$, which is defined as the ratio of the dynamic viscosity of the $i$ th branching FENE-P model (solute) to the zero-shear-rate viscosity of the solvent,

$$
\beta_{i}=\frac{\eta_{\mathrm{v} i}}{\eta_{\mathrm{N}}}
$$

From the above definition, the $\beta_{i}$ can be regarded as a dimensionless measurement of the solution concentration. The larger the $\beta_{i}$ is, the greater the concentration of solution is. Hereafter the unknown model parameters of the N-parallel FENE-P model turn to be $\lambda_{i}$ and $\beta_{i}$.

For the sake of concision but without loss of generality, the double-parallel FENE$\mathrm{P}$ constitutive model is taken as an example to illustrate the determination of unknown parameters of the proposed model. When the experimental data of apparent viscosity for polymer solution or surfactant solution is given, it is easily to get the zero-shearrate viscosity or apparent viscosity $\eta_{\alpha}$ with a certain initial shear rate. For the doubleparallel FENE-P constitutive model, the apparent viscosity is,

$$
\eta_{\alpha}=\eta_{\mathrm{N}}+\eta_{\mathrm{v} 1}+\eta_{\mathrm{v} 2}=\left(1+\beta_{1}+\beta_{2}\right) \eta_{\mathrm{N}}
$$

The above equation can also be expressed as,

$$
\beta_{1}+\beta_{2}=\eta_{\alpha} / \eta_{\mathrm{N}}-1
$$

In general, the experimental data of apparent viscosity and first normal stress difference can be measured by rheological experiment. Therefore, the values of apparent viscosity (or $\beta_{1}+\beta_{2}$ ) and first normal stress difference can be obtained for the doubleparallel FENE-P constitutive model, the unknown model parameters that need to be determined are $\beta_{1}$ (or $\beta_{2}$ ) and relaxation times $\lambda_{1}, \lambda_{2}$. Under this situation, the least square method can be utilized to determine the optimal unknown model parameters mentioned above. Similarly, the unknown parameters of the N-parallel FENE-P constitutive model can also be obtained through the least square method, but it needs more experimental data with the increase of $N$. 


\subsection{Validation and results discussion}

Figure 4 shows the comparison of N-parallel FENE-P constitutive model $(N=2)$ and traditional FENE-P constitutive model in describing the apparent viscosity of polymer solutions. In the figure, the independent experimental data of polymer solutions with different concentrations are chosen from the works of Ptasinski et al [11], Hashmet et al [12] and Pumode et al [13], respectively. It can be easily observed that the calculation results of the proposed model agree with the experimental data more accurate than the traditional model in the whole shear rate range $0.1 \mathrm{~s}^{-1} \sim 1000 \mathrm{~s}^{-1}$, the advantage is more remarkable especially when the shear rate is in the range of $10 \mathrm{~s}^{-1} \sim 1000 \mathrm{~s}^{-1}$. In addition, the N-parallel FENE-P model performs a favorable applicability for different concentrations of polymer solutions compared with the conventional model.

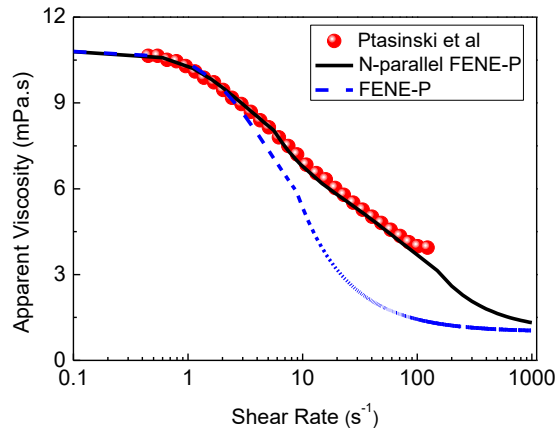

(a) N-parallel FENE-P: $N=2, \lambda_{1}=1, \lambda_{2}=25$, $\beta_{1}=4.3, \beta_{2}=5.5$; FENE-P: $\lambda=16, \beta=9.8$

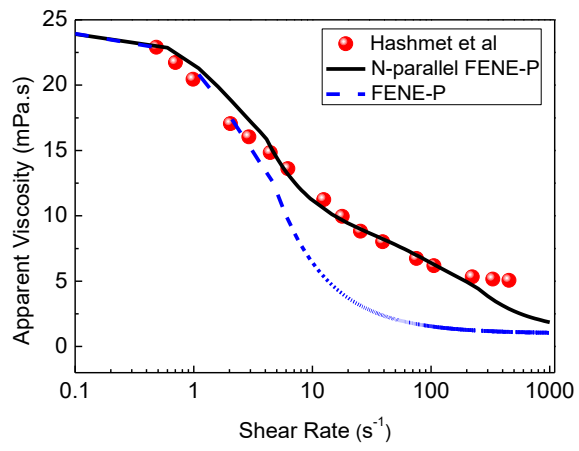

(b) N-parallel FENE-P: $N=2, \lambda_{1}=0.6, \lambda_{2}=35$, $\beta_{1}=7, \beta_{2}=16$; FENE-P: $\lambda=30, \beta=23$

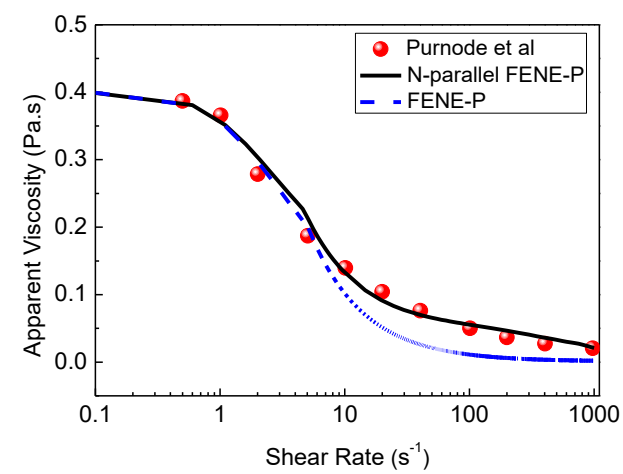

(c) N-parallel FENE-P: $N=2, \lambda_{1}=0.18, \lambda_{2}=30, \beta_{1}=49, \beta_{2}=350$; FENE-P: $\lambda=28, \beta=399$

Fig. 4. Comparison of N-parallel FENE-P constitutive model $(N=2)$ and traditional FENE-P model in describing the apparent viscosity of polymer solutions.

The main reason responsible for the advantages can be found from the basic idea of the N-parallel FENE-P model. From a microcosmic view, when $N$ branching FENE-P models are adopted in constitutive model, the relaxation-deformation of the microstructures formed in the polymer solutions can be controlled and modulated by small relaxation time and large relaxation time simultaneously. It can reflect the actual relaxation- 
deformation characteristics of the microstructures more truly and comprehensively. However, there is only single relaxation time in the traditional constitutive model, it can not represent the small relaxation-deformation and large relaxation-deformation of the microstructures at the same time, which is not consistent with the physical reality. Therefore, the proposed constitutive model is more reasonable than the conventional one. Correspondingly, the accuracy of the proposed constitutive model is much higher.

Besides, from Fig. 4 we can also find the adjustability of model parameters of the proposed model is much better than that of the traditional model. The apparent viscosity curves with different shear-thinning rates can be obtained by modulating the model parameters of the branching FENE-P models. The increase and decrease of the apparent viscosity curves are influenced more easily by the $\beta_{i}$, the slope of the apparent viscosity curves are affected more obviously by the $\lambda_{i}$.

Similar to Fig. 4, Fig. 5 demonstrates the comparative results of N-parallel FENE-P constitutive model $(N=2)$ and traditional model in describing the apparent viscosity of surfactant solutions (CTAC). In the figure the experimental data of surfactant solutions under different temperatures and concentrations are chosen from the studies of Zhang et al [14] and Qi et al [15], respectively. From Fig. 5, it is a pity to see that both the Nparallel FENE-P model and the traditional model can not able to represent the unique shear thickening behavior of surfactant solution. Because for both the N-parallel FENEP constitutive model and the traditional model, it can be proved that the FENE-P constitutive model is monotonic for the relation of apparent viscosity and share rate. The shear thickening phenomenon of the surfactant solution can be depicted by using a piecewise definition, such as the work of Galindo-Rosales[16]. However, it requires the introduction of at least 11 parameters to determine the piecewise constitutive model, which is nearly not practical in the engineering application. Fortunately, the proposed constitutive model in this paper still wins obvious advantage over the traditional one in shear rate range $10 \mathrm{~s}^{-1} \sim 1000 \mathrm{~s}^{-1}$. Furthermore, the N-parallel FENE-P model can describe all the apparent viscosities of surfactant solutions measured under different temperatures and concentrations, which indicates that the N-parallel FENE-P model has a much wider application.
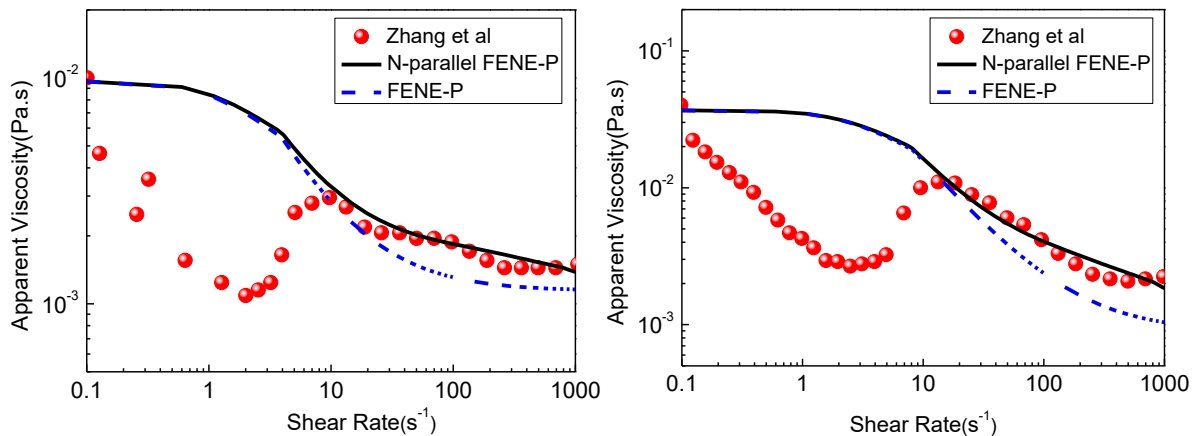

(a) $120 \mathrm{ppm}, 15^{\circ} \mathrm{C}, \eta_{\mathrm{N}}=1.1404 \times 10^{-3} \mathrm{~Pa} . \mathrm{s}(\mathrm{N}-$

(b) $300 \mathrm{ppm}, 25^{\circ} \mathrm{C}, \eta_{\mathrm{N}}=0.8937 \times 10^{-3} \mathrm{~Pa} . \mathrm{s}$ (N- 
parallel FENE-P: $N=2, \quad \lambda_{1}=0.18, \quad \lambda_{2}=35.6$, $\beta_{1}=0.5, \beta_{2}=7$; FENE-P: $\lambda=34.1, \beta=7.5$ )

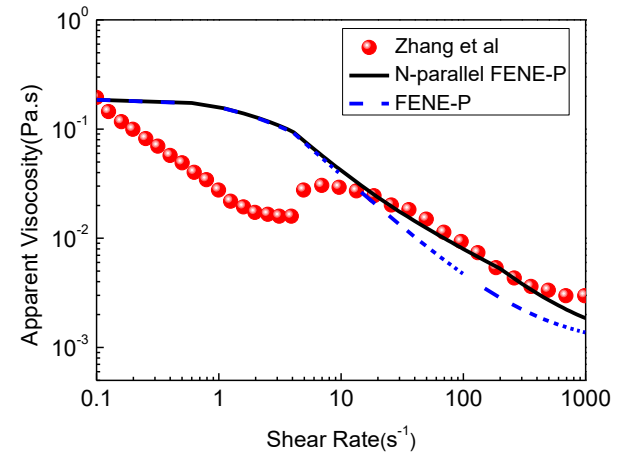

(c) $600 \mathrm{ppm}, 20^{\circ} \mathrm{C}, \eta_{\mathrm{N}}=1.0 \times 10^{-3} \mathrm{~Pa} . \mathrm{s}$ (N-parallel FENE-P: $N=2, \lambda_{1}=0.71, \lambda_{2}=35.6, \beta_{1}=5$,

$\beta_{2}=180$; FENE-P: $\lambda=35.2, \beta=185$ ) parallel FENE-P: $N=2, \quad \lambda_{1}=0.18, \quad \lambda_{2}=17.8$, $\beta_{1}=2, \beta_{2}=38$; FENE-P: $\lambda=17.1, \beta=40$ )

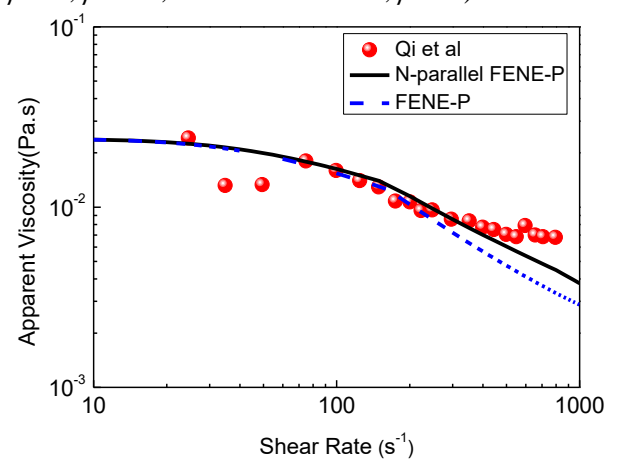

(d) $(5 \mathrm{mM} / 5 \mathrm{mM}), 20^{\circ} \mathrm{C}, \eta_{\mathrm{N}}=1.0 \times 10^{-3} \mathrm{~Pa} . \mathrm{s}$ (N-parallel FENE-P: $N=2, \lambda_{1}=0.18, \lambda_{2}=0.89$, $\beta_{1}=3, \beta_{2}=20$; FENE-P: $\lambda=0.87, \beta=23$ )

Fig. 5. Comparison of $N$-parallel FENE-P constitutive model $(N=2)$ and traditional FENE-P model in describing the apparent viscosity of surfactant solutions.

The normal stress difference of surfactant solutions is not equal to zero because surfactant solution is a type of non-Newtonian fluids. Here the comparison of the first normal stress difference $\left(N_{1}(\dot{\gamma})=\tau_{x x}-\tau_{y y}\right)$ is illustrated in Fig. 6. The experimental data set are selected from the work of Qi et al [15], in which the first normal stress difference of surfactant solution Arquad 16-50/NaSal was given in detail. Fig.6 indicates that the calculated first normal stress difference of the proposed constitutive model provides an excellent fit to the experimental data, the proposed model can represent the first normal stress difference more accurate than the traditional model. Especially when the shear rate is larger than $150 \mathrm{~s}^{-1}$, the traditional model can't depict the first normal stress difference accurately compare to the experimental data, the maximum relative error can up to $35 \%$.

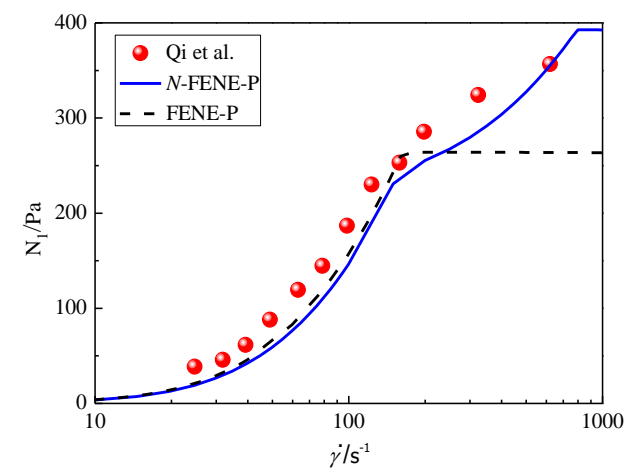

$5 \mathrm{mM} / 5 \mathrm{mM}, 20^{\circ} \mathrm{C}, \eta_{\mathrm{N}}=1.0 \times 10^{-3} \mathrm{~Pa} . \mathrm{s}\left(\mathrm{N}-\right.$ parallel FENE-P: $N=2, \lambda_{1}=0.18, \lambda_{2}=0.89, \beta_{1}=3$, $\beta_{2}=20$; FENE-P: $\lambda=0.87, \beta=23$ ) 
Fig. 6. Comparison of N-parallel FENE-P constitutive model $(N=2)$ and traditional FENE-P model in describing the first normal stress difference of surfactant solution

It is worth pointing out that for the proposed N-parallel FENE-P model, although the adjustability of the model parameters becomes better and the accuracy of the calculation results becomes higher with the increase of branching number $N$, the numerical workload also becomes heavier following the similar tendency. Therefore, it is significant to take both the computational accuracy and workload into consideration to choose the branching number. $N=2$ and $N=3$ were adopted in our numerical experiment and it was found that the calculation accuracy of the apparent viscosity when $N=3$ improved slightly compared with that when $N=2$. The calculation accuracy of the apparent viscosity and first normal stress difference has already been much higher than that of the traditional model when $N=2$, this is the reason why we only present the comparative results when $N=2$ in this Section.

\section{Conclusions}

The present study focuses on the constitutive model describing the apparent viscosity of viscoelastic fluids and an N-parallel FENE-P constitutive model is proposed based on multiple relaxation times. From our study, the following conclusions can be summarized:

(1) The proposed N-parallel FENE-P model is a more general constitutive model with better applicability. It utilizes the $N$ branching FENE-P models to describe the rheological behaviors of viscoelastic fluid and characterize the anisotropy of relaxationdeformations of the microstructures formed in viscoelastic fluid, which is more consistent with the real physical process.

(2) Compared with the traditional FENE-P model, the proposed model demonstrates a favorable adjustability of the model parameters. The apparent viscosity curves with different shear thinning rates can be obtained by modulating the model parameters of the branching FENE-P models.

(3) The proposed N-parallel FENE-P model is shown to perform an excellent fit to several independent experimental data sets. It can represent the apparent viscosity of polymer solution more accurate than the traditional model in whole shear rate range $0.1 \mathrm{~s}^{-1} \sim 1000 \mathrm{~s}^{-1}$, the advantage is more remarkable especially when the shear rate is among $10 \mathrm{~s}^{-1} \sim 1000 \mathrm{~s}^{-1}$. Although the proposed N-parallel FENE-P model can also not capture the shear thickening behavior of surfactant solution as the conventional model, it still possesses advantages over the traditional model in representing the apparent viscosity and first normal stress difference within the shear rate range $10 \mathrm{~s}^{-1} \sim 1000 \mathrm{~s}^{-1}$.

\section{Acknowledgements}

The authors thank for support of National Natural Science Foundation of China (No. 51636006), project of Construction of Innovative Teams and Teacher Career Development for Universities and Colleges under Beijing Municipality (No. IDHT20170507), 
National Key R\&D Program of China (Grant No. 2016YFE0204200) and the Program of Great Wall Scholar (CIT\&TCD20180313).

\section{References}

1. Toms B. A.: Some observations on the flow of linear polymer solutions through straight tubes at large Reynolds numbers. Proceedings of the 1st International Rheology Congress, II, Part 2, pp. 135-142, North Holland Publish Co., Netherlands (1949).

2. Renardy M., Renardy Y.: Linear stability of plane Couette flow of an upper convected Maxwell fluid. Journal of Non-Newtonian Fluid Mechanics 22, 23-33 (1986).

3. Oldroyd J. G.: On the formulation of rheological equations of state. Proceedings of the Royal Society A 200, 523-541 (1950).

4. Oliveria P. G.: Alternative derivation of differential constitutive equations of the OldroydB type. Journal of Non-Newtonian Fluid Mechanics 160, 40-46 (2009).

5. Giesekus H.: A simple constitutive equation for polymer fluids based on the concept of deformation-dependent tensorial mobility. Journal of Non-Newtonian Fluid Mechanics 11, 69109 (1982).

6. Bird R. B., Dotson P. J., Johnson N. L.: Polymer solution rheology based on a finitely extensible bead-spring chain model. Journal of Non-Newtonian Fluid Mechanics 7, (2-3): 213235 (1980).

7. Everaers R., Sukumaran S. K., Grest G. S., Svaneborg C., Sivasubramanian A., Kremer K.: Rheology and microscopic topology of entangled polymeric liquids. Sciences 303, 5659: 823-826 (2004).

8. Ezrahi S., Tuval E., Aserin A.: Properties, main applications and perspectives of worm micelles. Advances in Colloid and Interface Science 128-130, 77-102 (2006).

9. Peterlin A.: Streaming birefringence of soft linear macromolecules with finite chain length. Polymer 2, 257-264 (1961).

10. Wei J. J., Yao Z. Q.: Rheological characteristic of drag reducing surfactant solution. Journal of Chemical Industry and Engineering (Chinese) 58, 2: 0335-0340 (2007).

11. Ptasinski P. K., Nieuwstadt F. T. M., Van Den Brule B. H. A. A., Hulsen M. A.: Experiments in turbulent pipe flow with polymer additives at maximum drag reduction. Flow, Turbulence and Combustion 66, 159-182 (2001).

12. Hashmet M. R., Onur M., Tan I. M.: Empirical correlations for viscosity of polyacrylamide solutions with the effects of concentration, molecular weight and degree of hydrolysis of polymer. Journal of Applied Sciences, 14, 10: 1000-1007 (2014).

13. Purnode B., Crochet M. J.: Polymer solution characterization with the FENE-P model. Journal of Non-Newtonian Fluid Mechanics 77, 1-20 (1998).

14. Zhang H. X., Wang D. Z., Gu W. G., Chen H. P.: Effects of temperature and concentration on rheological characteristics of surfactant additive solutions. Journal of Hydrodynamics 20, 5: 603-610 (2008).

15. Qi Y. Y., Littrell K., Thiyagarajan P., Talmon Y., Schmidt J., Lin Z. Q.: Small-angle neutron scattering study of shearing effects on drag-reducing surfactant solutions. Journal of Colloid and Interface Science 337, 218-226 (2009).

16. Galindo-Rosalesa F. J., Rubio-Hernández F. J., Sevilla A.: An apparent viscosity function for shear thickening fluids. Journal of Non-Newtonian Fluid Mechanics 166, 321-325 (2011). 